\section{Treinamento esfincteriano precoce: prevalência, características materna da criança e fatores associados numa coorte de nascimentos}

\author{
Early toilet training: prevalence, the \\ characteristics of the mother and of the \\ child and associated factors in a cohort of \\ births
}

\author{
Denise Marques Mota 1 \\ Aluisio Jardim Dornellas Barros 2
}

1,2 Programa de Pós-graduação em Epidemiologia. Departamento de Medicina-Social. Faculdade de Medicina. Universidade Federal de Pelotas. Marechal Deodoro, 1160. Pelotas, RS, Brasil. CEP 96.020-220. E-mail: denise.sul@brturbo.com.br

\begin{abstract}
Objectives: to assess the prevalence of children not using diapers, the age at which toilet training started and the mother's expectations regarding the attainment of this in a cohort of births.

Methods: all children born in 2004 in the city of Pelotas, in the State of Rio Grande do Sul, Brazil, were enrolled in a longitudinal study. At the age of 12 months they were assessed for their development. Mothers were questioned regarding the beginning of toilet training and parental expectations in relation to the age at which daytime toilet training should be completed. Differences between groups were assessed using chi-squared tests for heterogeneity and linear tendency.

Results: at 12 months of age $14.7 \%$ of the mothers had begun daytime toilet training. Only $2.2 \%$ of the mothers were provided guidance by pediatricians regarding cessation of diaper use. The groups of mothers with the higher prevalence of starting toilet training at 12 months were the ones from the top economic quintile, five to eight years of schooling, adolescent mothers and mothers aged over 40 years. Two thirds of the mothers believed the ideal time to cease using diapers is before 18 months of age; $1.3 \%$ of children did not use diapers during the day.

Conclusions: toilet training began early in a significant number of children and an insignificant proportion of mothers received guidance from pediatricians on this. Information regarding the ideal time and adequate methods for introducing sphincter control should be offered to mothers during puerperium as part of basic health care.
\end{abstract}

Key words Toilet training, Elimination disorders, Urinary tract infections, Enuresis, Children

\section{Resumo}

Objetivos: avaliar a prevalência de crianças sem fraldas, a idade de início do treinamento esfincteriano $e$ a expectativa materna em relação à aquisição deste controle numa coorte de nascimentos.

Métodos: todas as crianças nascidas em $2004 \mathrm{em}$ Pelotas, Rio Grande do Sul, Brasil, foram incluídas em um estudo longitudinal. Aos 12 meses, as crianças tiveram seu desenvolvimento avaliado e as mães questionadas sobre o inicio do treinamento esfincteriano $e$ expectativa em relação à idade da retirada das fraldas. Diferenças entre grupos foram avaliadas através de testes qui-quadrado para heterogeneidade e tendência linear.

Resultados: aos 12 meses de idade, 14,7\% das mães tinham iniciado o treinamento esfincteriano. Apenas $2,2 \%$ receberam orientação pediátrica sobre retirada de fraldas. Os grupos de mães com maior prevalência de início de treinamento aos 12 meses foram as do primeiro quintil economico, cinco a oito anos de escolaridade, adolescentes e maiores de 40 anos. Dois terços acham que o momento para deixar as fraldas é antes dos 18 meses; 1,3\% das crianças estão sem fraldas de dia.

Conclusões: o treinamento esfincteriano começou precocemente em uma parcela significativa destas crianças, sendo desprezivel a proporção de mães orientadas pelos pediatras. Informações sobre o momento ideal e métodos adequados de controle esfincteriano devem ser oferecidas às mães, no contexto da puericultura e atenção básica à saúde.

Palavras-chave Treinamento no uso de toalete, Transtornos da excreção, Infecção urinária, Enurese, Crianças 


\section{Introdução}

O aprendizado do controle esfincteriano, um dos marcos do desenvolvimento infantil, é um processo evolutivo encontrado em todas as crianças, existindo muita variabilidade individual na idade de aquisição - peso de nascimento, sexo, raça, diferenças culturais, dessa habilidade. 1-4

A idade, isoladamente, não é um bom indicador do preparo de uma criança para começar o treinamento esfincteriano. A criança deve apresentar algumas habilidades para iniciar esse processo, como sentar, levantar e caminhar para poder dirigirse ao banheiro; falar para demonstrar suas necessidades; entender e seguir instruções para ser ensinada; tirar a roupa para evacuar e urinar em local adequado. Essas habilidades encontram-se presentes na maioria das crianças por volta dos 18 meses. 5-6

Até os anos 30 a atitude predominante para o treinamento de esfíncteres era a da permissividade, mudando para a rigidez, quando os pais eram aconselhados a treinar seus filhos o mais cedo possível, a fim de liberá-los da obrigação de mudar as fraldas. 7 Com o evoluir das evidências, alguns clínicos concluíram que o desenvolvimento do controle urinário e fecal é um processo de maturação que não deve ser acelerado pelo treinamento esfincteriano e sim deixado até que a criança mostre desejo de ser treinada. 8

Um modelo de treinamento para controle de esfíncteres foi proposto por Brazelton, ${ }^{9}$ em 1962, sendo ainda um guia nos dias de hoje. Esse modelo fundamenta-se na orientação "passiva" que além da maturação fisiológica da criança, considera o interesse e aptidão psicossocial para iniciar o treinamento, tendo sido elaborado com a finalidade de minimizar o conflito e a ansiedade e ressaltar a importância da flexibilidade. O treinamento deve ser conduzido de maneira relativamente suave e tendose a confiança de que a criança aprenderá a ir sozinha ao banheiro, no tempo certo.

O processo de retirada de fraldas precoce, tardio ou mal conduzido é um determinante no aparecimento da síndrome de disfunção das eliminações, que consiste em disfunções relativas ao aparelho urinário e gastrintestinal. 10-12 Crianças com treinamento precoce apresentam, com maior freqüência, infecção do trato urinário, constipação e sintomas de disfunção miccional (incontinência urinária, manobras de contenção, enurese, urgência miccional). 13,14 Os programas de tratamento para disfunção miccional utilizam técnicas de retreinamento do controle vesical, que, nada mais são, do que as técnicas utilizadas há 40 anos atrás para o treinamento do controle esfincteriano em crianças. 15 Estes achados reforçam a hipótese dos autores deste trabalho de que os freqüentes problemas de disfunção miccional possam ser causados por estratégias inadequadas de treinamento usados mais recentemente e que, a aquisição da capacidade vesical adequada, no tempo correto, pode evitar a instabilidade vesical tardia. 16

A literatura atual é contraditória em relação a como e quando treinar a criança. Informações atualizadas são necessárias porque as referências citadas sobre treinamento esfincteriano são de décadas passadas e inexistentes na população brasileira.

O objetivo deste estudo foi analisar, na visita de 12 meses da coorte de nascimentos de Pelotas, em 2004, as prevalências das crianças sem fraldas durante o dia e durante a noite, a idade em que as mães iniciaram o treinamento esfincteriano e avaliar as expectativas delas em relação à aquisição deste controle, assim como, a origem da informação sobre treinamento esfincteriano. As características maternas e das crianças já em treinamento, assim como os fatores associados ao início do treinamento também foram estudados.

\section{Métodos}

Este estudo foi realizado no contexto de uma coorte de nascimentos iniciada em 2004 no município de Pelotas, Rio Grande do Sul. Nesse ano, as cinco maternidades da cidade foram visitadas diariamente e todas as mães dos 4263 nascidos vivos, residentes na área urbana da cidade foram recrutadas para o estudo. As mães foram entrevistadas durante a hospitalização e seus bebês medidos (comprimento e peso) e examinados para avaliação da idade gestacional.

As crianças foram novamente procuradas ao completarem 12 meses, sendo realizada entrevista com as mães e avaliação antropométrica das crianças. Os detalhes dos métodos da Coorte de Nascimentos de Pelotas, 2004, estão disponíveis em outra publicação. ${ }^{17}$

$\mathrm{Na}$ visita dos 12 meses, as mães responderam, em suas casas, um questionário sobre condições de saúde da criança, morbidades, alimentação, padrões de sono e vacinação, assim como dados relativos à saúde materna. Foi feito um levantamento detalhado sobre o treinamento de esfíncteres, incluindo idade em meses do início do treinamento esfincteriano, uso ou não de fraldas durante o dia ou noite, idade de retirada das fraldas de dia ou noite em meses, idade em que a mãe acha mais adequado iniciar a retirada 
das fraldas, recebimento de orientação sobre como proceder para retirar as fraldas, hábito intestinal da criança e uso de laxativos no último mês. Foram realizadas medidas antropométricas (peso, comprimento, perímetro abdominal e cefálico) e aplicou-se a versão de rastreamento do teste de desenvolvimento de Battelle, segundo Newborg et al. 18 e itens selecionados do teste de desenvolvimento de Denver citado por Frankenburg et al. ${ }^{19}$

As entrevistadoras foram treinadas por um pediatra especialista em desenvolvimento infantil e acompanhadas regularmente durante as visitas e aplicação do teste nas crianças por outro pediatra. O controle de qualidade incluiu a repetição de $10 \%$ das entrevistas no domicílio, utilizando um questionário reduzido. Um contato pelo telefone foi tentado com todas as mães não incluídas no grupo anterior e que dispunham de telefone em casa. Quinzenalmente todas as entrevistadoras eram acompanhadas por um supervisor de pesquisa com objetivo de avaliar a aplicação do teste de desenvolvimento e do questionário.

Os desfechos estudados foram: idade em meses do início do treinamento de esfíncteres, uso de fraldas diurno e noturno, expectativa materna em relação à idade de retirada de fraldas e recebimento de orientação médica sobre controle de esfíncteres.

As demais variáveis incluídas nas análises foram: sexo da criança; características maternas: escolaridade, idade em anos completos, paridade e trabalho materno; características do domicílio: número de crianças na residência e nível econômico em quintis de referência para Pelotas do Indicador Econômico Nacional (IEN). 20

As habilidades necessárias para a aquisição do controle urinário e fecal foram avaliadas a partir dos testes de desenvolvimento de Battelle e Denver, ambos citados por Newborg et al. ${ }^{18}$, e Frankenburg et al. ${ }^{19}$ respectivamente, sendo utilizados para este estudo os itens relativos a sentar (consegue sentar quando está engatinhando ou caminhando), caminhar bem (com firmeza, caindo pouco e não se balançando para os lados), falar (mais de dez palavras), receber ordens (seguir duas ou mais ordens entre quatro solicitadas) e tirar a roupa (tirar uma peça de roupa, com ou sem ajuda, exceto sapatos e meias).

A análise foi realizada através do programa Stata 9.0, 2005. Foram utilizados os testes qui-quadrado para comparar prevalências conforme exposições dicotômicas e qui-quadrado de tendência linear para exposições ordinais. Optou-se pela estratificação por sexo para análise dos desfechos esfincterianos devido aos relatos de literatura sobre diferenças entre os sexos na aquisição do controle esfincteriano.

O protocolo do estudo foi aprovado pela Comissão de Ética e Pesquisa da Faculdade de Medicina da Universidade Federal de Pelotas. Foi solicitado o consentimento escrito para a participação no estudo, após a mãe ter sido informada sobre os objetivos e ter garantida a confidencialidade das informações. O trabalho de campo ocorreu de dezembro de 2004 a dezembro de 2005.

\section{Resultados}

Em 2004 nasceram vivas 4263 crianças residentes na zona urbana da cidade de Pelotas, sendo incluídas 4231 no estudo perinatal ( $0,8 \%$ perdas e recusas). Durante o primeiro ano de vida morreram 82 crianças, ficando elegíveis para esta visita dos 12 meses 4149 crianças. Destas crianças tivemos 216 perdas e 26 recusas, restando $91,6 \%$ da amostra original. Para este estudo foram excluídas sete crianças com meningomielocele, duas com paralisia cerebral e 36 gemelares, resultando numa amostra de 3862 crianças. As perdas foram devidas à mudança de cidade em sua grande maioria, com poucas recusas $(0,6 \%)$.

Das crianças recrutadas para o acompanhamento $51,8 \%$ eram do sexo masculino, $14,2 \%$ prematuros (idade gestacional $<37$ semanas) e $8,3 \%$ com baixo peso ao nascer $(<2500 \mathrm{~g})$.

A maior parte das mães se encontrava na faixa etária de 20 a 29 anos, com média de idade de 26,2 anos (desvio-padrão=6,8) e uma proporção de $18,7 \%$ de mães adolescentes. A média de anos de estudo foi de 8,2 anos (desvio-padrão=3,5), sendo que o maior grupo apresentava escolaridade entre cinco e oito anos. Cerca de $40 \%$ eram primigestas. A Tabela 1 descreve as características das mães e das crianças, assim como características socioeconômicas da amostra.

Observou-se concentração das mães no primeiro quintil de classificação do Índice Econômico Nacional (IEN), revelando a maior fecundidade entre os estratos mais pobres da população.

Para análise das variáveis relacionadas com o desenvolvimento infantil e controle esfincteriano foi feita uma estratificação por sexo. (Tabela 2)

Apenas 9,4\% das crianças apresentavam quatro ou mais habilidades necessárias para o adequado aprendizado do controle esfincteriano. As habilidades mais freqüentes entre as crianças foram: sentar sozinho $(95,3 \%)$ e tirar uma peça de roupa $(42,8 \%)$. As demais habilidades estiveram presentes em menos $40 \%$ das crianças. Quando perguntadas sobre 
Características perinatais e socioeconômicas das 3862 crianças nascidas de parto único, pertencentes à coorte de nascimento 2004. Pelotas, Rio Grande do Sul.

\begin{tabular}{|c|c|c|}
\hline Variável & $\mathbf{N}$ & $(\%)$ \\
\hline \multicolumn{3}{|l|}{ Idade gestacional (semanas) $(n=3858)$} \\
\hline Pré-termo $(\leq 36)$ & 546 & 14,2 \\
\hline A termo $(37-41)$ & 3068 & 79,5 \\
\hline Pós-termo $(\geq 42)$ & 244 & 6,3 \\
\hline Peso ao nascer $<2500$ gramas $(n=3869)$ & 321 & 8,3 \\
\hline Complicações perinatais $(n=3861)$ & 282 & 7,3 \\
\hline \multicolumn{3}{|l|}{ Asfixia neonatal } \\
\hline (apgar $\leq 3$ no $5 \circ$ minuto) $(n=3850)$ & 6 & 0,2 \\
\hline \multicolumn{3}{|l|}{ Número de crianças em casa $(n=3861)$} \\
\hline 1 & 1812 & 46,9 \\
\hline 2 & 1105 & 28,6 \\
\hline$\geq 3$ & 944 & 24,5 \\
\hline \multicolumn{3}{|l|}{ Idade materna (anos) $(n=3859)$} \\
\hline Adolescente $(\leq 19)$ & 720 & 18,7 \\
\hline $20-29$ & 1915 & 49,6 \\
\hline $30-39$ & 1098 & 28,5 \\
\hline$\geq 40$ & 126 & 3,3 \\
\hline \multicolumn{3}{|l|}{ Escolaridade materna (anos) $(n=3823)$} \\
\hline $0-4$ & 582 & 15,2 \\
\hline $5-8$ & 1569 & 41,0 \\
\hline-11 & 1282 & 33,5 \\
\hline$\geq 12$ & 390 & 10,2 \\
\hline \multicolumn{3}{|l|}{ Paridade $(n=3860)$} \\
\hline 1 & 1529 & 39,6 \\
\hline 2 & 1006 & 26,1 \\
\hline 3 & 626 & 16,2 \\
\hline$\geq 4$ & 699 & 18,1 \\
\hline \multicolumn{3}{|l|}{ Classificação socioeconômica $(n=3860)$} \\
\hline \multicolumn{3}{|l|}{ Quintis do Indicador Econômico Nacional } \\
\hline 1 (20\% mais pobres) & 897 & 23,3 \\
\hline 2 & 799 & 20,7 \\
\hline 3 & 849 & 21,9 \\
\hline 4 & 704 & 18,3 \\
\hline 5 & 611 & 15,9 \\
\hline \multicolumn{3}{|l|}{ Mãe trabalha fora de casa } \\
\hline Sim & 1522 & 39,4 \\
\hline Não & 2336 & 60,6 \\
\hline
\end{tabular}


Prevalência e distribuição, quanto ao sexo, das habilidades relacionadas ao controle esfincteriano e dos desfechos aos 12 meses de idade, nas 3862 crianças nascidas de parto único pertencentes à coorte de nascimentos 2004. Pelotas, Rio Grande do Sul.

\begin{tabular}{|c|c|c|c|c|c|}
\hline \multirow[t]{2}{*}{ Variáveis } & \multirow{2}{*}{$\begin{array}{c}\begin{array}{c}\text { Masculino } \\
(n=2001)\end{array} \\
\%\end{array}$} & \multirow{2}{*}{$\begin{array}{c}\begin{array}{c}\text { Feminino } \\
(n=1861)\end{array} \\
\%\end{array}$} & \multirow{2}{*}{$\frac{\begin{array}{c}\text { Todos } \\
(n=3862)\end{array}}{\%}$} & \multirow[t]{2}{*}{$\chi^{2}$} & \multirow[t]{2}{*}{ Valor $p$} \\
\hline & & & & & \\
\hline Consegue sentar quando está de pé ou engatinhando & 95,6 & 95,0 & 95,3 & 0,35 & 0,554 \\
\hline Caminha bem, com firmeza & 23,1 & 23,3 & 23,2 & 0,04 & 0,847 \\
\hline Tira uma peça de roupa com ou sem ajuda & 41,0 & 44,9 & 42,8 & 6,22 & 0,013 \\
\hline \multicolumn{6}{|l|}{ Número de ordens seguidas entre quatro solicitadas } \\
\hline$<2$ & 39,1 & 30,5 & 34,9 & 38,33 & $<0,001$ \\
\hline 2 & 29,0 & 29,8 & 29,4 & & \\
\hline$\geq 3$ & 32,0 & 39,8 & 35,7 & & \\
\hline Fala mais de dez palavras & 2,7 & 5,0 & 3,8 & 13,96 & $<0,001$ \\
\hline \multicolumn{6}{|l|}{ Número de habilidades presentesł } \\
\hline $0-1$ & 20,4 & 16,2 & 18,4 & 24,85 & $<0,001$ \\
\hline 2 & 42,4 & 39,8 & 41,2 & & \\
\hline 3 & 29,3 & 33,0 & 31,1 & & \\
\hline$\geq 4$ & 8,0 & 11,0 & 9,4 & & \\
\hline Iniciou treinamento esfincteriano & 13,6 & 15,9 & 14,7 & 4,15 & 0,042 \\
\hline Sem fraldas de dia & 1,4 & 1,2 & 1,3 & 0,19 & 0,659 \\
\hline Sem fraldas à noite & 0,1 & 0,1 & 0,1 & 0,00 & 0,959 \\
\hline Recebeu orientação médica sobre treinamento & 2,1 & 2,3 & 2,2 & 12,61 & 0,082 \\
\hline \multicolumn{6}{|c|}{ Expectativa materna sobre idade adequada para retirar fraldas (meses) } \\
\hline$\leq 12$ & 15,2 & 19,0 & 17,0 & 14,2 & $<0,001$ \\
\hline $13-18$ & 51,2 & 48,7 & 50,0 & & \\
\hline $19-24$ & 24,6 & 24,6 & 24,6 & & \\
\hline$\geq 24$ & 2,2 & 1,3 & 1,8 & & \\
\hline Não sabem & 1,3 & 0,7 & 1,0 & & \\
\hline De acordo com habilidades* & 5,6 & 5,6 & 5,6 & & \\
\hline
\end{tabular}

‡ Habilidades: sentar, caminhar bem, tirar a roupa com ou sem ajuda, falar mais de dez palavras, seguir duas ou mais instruções entre quatro solicitadas; * Mães que não responderam idade que acham adequado iniciar o treinamento e sim habilidades da criança (caminhar, falar, querer retirar as fraldas)

qual acreditavam ser a idade mais adequada para retirar as fraldas da criança, a maioria das mães $(67 \%)$ relatou esperar que seus filhos estivessem treinados até os 18 meses de idade. Uma pequena parcela $(5,6 \%)$, em vez de referir uma idade, usou a presença de alguma habilidade (por exemplo, caminhar, falar) como parâmetro para o início do treinamento.

Nas crianças que já tinham iniciado o treinamento esfincteriano apenas $16,9 \%$ tinham quatro ou 
Indicadores de treinamento esfincteriano de acordo com o nível socioeconômico aos 12 meses de idade, nas 3860* crianças nascidas de parto único pertencentes à coorte de nascimentos 2004. Pelotas, Rio Grande do Sul.

\begin{tabular}{|c|c|c|c|c|c|c|c|}
\hline \multirow[t]{2}{*}{ Variável } & \multicolumn{7}{|c|}{ Nível socioeconômico (Quintis do Indicador Econômico Nacional, Pelotas) } \\
\hline & $\begin{array}{c}1 \\
(n=897)\end{array}$ & $\begin{array}{c}2 \\
(n=799)\end{array}$ & $\begin{array}{c}3 \\
(n=849)\end{array}$ & $\begin{array}{c}4 \\
(n=704)\end{array}$ & $\begin{array}{c}5 \\
(n=611)\end{array}$ & $\chi^{2}$ & Valor $p$ \\
\hline Iniciou treinamento esfincteriano & 16,7 & 14,5 & 15,2 & 15,5 & 10,3 & 7,40 & $<0,001 \mp$ \\
\hline Sem fraldas de dia & 2,6 & 1,1 & 1,0 & 0,9 & 0,8 & 9,42 & $<0,001 \neq$ \\
\hline \multicolumn{6}{|c|}{ Expectativa materna da idade de controle esfincteriano (meses) } & 82,11 & $<0,001 \dagger$ \\
\hline$\leq 12$ & 19,4 & 18,8 & 19,8 & 15,1 & 9,7 & & \\
\hline $13-18$ & 48,6 & 50,3 & 51,4 & 54,6 & 44,7 & & \\
\hline $19-24$ & 23,1 & 23,4 & 21,2 & 23,0 & 34,9 & & \\
\hline$\geq 24$ & 2,7 & 1,3 & 1,2 & 1,6 & 2,3 & & \\
\hline De acordo com habilidades§ & 5,4 & 5,9 & 5,9 & 4,4 & 6,6 & & \\
\hline Não sabem & 0,9 & 0,4 & 0,6 & 1,4 & 2,0 & & \\
\hline Recebeu orientação médica & 1,0 & 1,6 & 1,8 & 1,9 & 5,6 & 75,15 & $<0,001 \dagger$ \\
\hline \multicolumn{8}{|l|}{ Presença de habilidades } \\
\hline \multicolumn{8}{|l|}{ Consegue sentar quando de pé } \\
\hline ou engatinhando & 92,6 & 94,5 & 96,7 & 96,2 & 97,4 & 22,00 & $<0,001 \neq$ \\
\hline Caminhar bem firme & 17,0 & 22,3 & 25,2 & 25,0 & 28,5 & 29,27 & $<0,001 \neq$ \\
\hline Falar mais de dez palavras & 2,5 & 4,6 & 5,4 & 3,1 & 3,1 & 14,60 & $<0,001 \dagger$ \\
\hline \multicolumn{8}{|l|}{ Seguir mais de duas ordens } \\
\hline entre quatro solicitadas & 57,3 & 64,1 & 65,2 & 70,6 & 71,4 & 41,99 & $<0,001 \neq$ \\
\hline Tirar uma peça de roupa com ou sem ajuda & 44,2 & 40,3 & 43,9 & 46,2 & 39,1 & 9,97 & $0,04 \dagger$ \\
\hline
\end{tabular}

* Duas crianças sem informação de renda; \pm Primeiro quintil de referência corresponde aos $20 \%$ mais pobres; § mães que não responderam idade que acham adequado iniciar o treinamento e citaram habilidades da criança (caminhar, falar, querer retirar as fraldas); $\dagger$ teste do qui-quadrado para associação; ₹ teste de tendência linear.

mais habilidades presentes. As habilidades mais freqüentes foram o sentar $(96,4 \%)$ e receber ordens $(78,4 \%)$. Nas crianças sem uso de fraldas durante o dia, $20 \%$ tinham quatro ou mais habilidades, sendo que sentar $(95 \%)$ e receber ordens $(78,4 \%)$ foram também as mais freqüentes. Observou-se que as meninas apresentaram um número maior de habilidades no início do treinamento esfincteriano em comparação com os meninos. As diferenças foram no seguir ordens quando solicitadas, falar e tirar a roupa.

A presença de habilidades também demonstra diferenças entre os níveis socioeconômicos (Tabela 3). A aquisição da linguagem foi mais freqüente entre as crianças dos quintis dois e três; retirar a roupa foi mais freqüente nas crianças do quintil quatro e um; as demais habilidades mostraram uma tendência de aumento de suas freqüências à medida que aumentava o nível socioeconômico.

O início do treinamento esfincteriano foi mais freqüente nas meninas e as mães de meninas apresentaram uma expectativa mais precoce em relação a largar as fraldas, assim como as mães mais pobres.

O nível socioeconômico influenciou a idade em que as mães iniciaram o treinamento esfincteriano, observando-se (Tabela 3 ) uma tendência em relação a um maior número de mães pobres iniciarem o treinamento mais cedo e seus filhos largarem as fraldas mais cedo.

Analisando características maternas (Tabela 4) 
Características maternas e treinamento esfincteriano nas 3862 crianças nascidas de parto único pertencentes à coorte de nascimentos 2004. Pelotas, Rio Grande do Sul.

\begin{tabular}{|c|c|c|c|c|c|c|c|}
\hline \multirow[t]{2}{*}{ Variáveis } & \multirow[t]{2}{*}{$\mathbf{N}$} & \multicolumn{3}{|c|}{ Iniciou treinamento diurno } & \multicolumn{3}{|c|}{ Sem fraldas de dia } \\
\hline & & $\%$ & $\chi^{2}$ & Valor $p$ & $\%$ & $\chi^{2}$ & Valor $p$ \\
\hline \multicolumn{8}{|l|}{ Paridade } \\
\hline 1 & 1529 & 15,3 & 0,48 & $0,50 *$ & 0,7 & 10,96 & $<0,001$ * \\
\hline 2 & 1006 & 14,0 & & & 1,4 & & \\
\hline 3 & 626 & 15,3 & & & 1,8 & & \\
\hline$\geq 4$ & 699 & 13,9 & & & 2,3 & & \\
\hline \multicolumn{8}{|c|}{ Escolaridade mãe (anos) } \\
\hline $0-4$ & 582 & 13,1 & 12,06 & $<0,001 \dagger$ & 2,9 & 19,10 & $<0,001$ * \\
\hline $5-8$ & 1569 & 16,5 & & & 1,5 & & \\
\hline $9-11$ & 1282 & 14,9 & & & 0,8 & & \\
\hline$\geq 12$ & 390 & 10,0 & & & 0,0 & & \\
\hline \multicolumn{8}{|l|}{ Idade mãe (anos) } \\
\hline Adolescente $(<19)$ & 720 & 17,4 & 5,50 & $0,15+$ & 1,7 & 2,27 & $0,23+$ \\
\hline $20-29$ & 1915 & 14,2 & & & 1,3 & & \\
\hline $30-39$ & 1098 & 13,8 & & & 1,1 & & \\
\hline$\geq 40$ & 126 & 15,9 & & & 2,4 & & \\
\hline \multicolumn{8}{|l|}{ Trabalho materno } \\
\hline Sim & 1522 & 13,4 & 3,45 & $0,06 \dagger$ & 1,1 & 1,40 & $0,23 \dagger$ \\
\hline Não & 2336 & 15,6 & & & 1,5 & & \\
\hline
\end{tabular}

† Qui-quadrado de associação; * Tendência linear.

Tabela 5

Características maternas e expectativa materna em relação à idade de controle esfincteriano nas 3862 crianças nascidas de parto único pertencentes à coorte de nascimentos 2004. Pelotas, Rio Grande do Sul.

\begin{tabular}{|c|c|c|c|c|c|c|c|c|c|}
\hline \multirow[t]{2}{*}{ Variáveis } & \multirow[t]{2}{*}{$\mathrm{N}$} & \multicolumn{6}{|c|}{ Expectativa materna idade retirar fraldas (meses) } & \multirow[t]{2}{*}{$\chi^{2}$} & \multirow[t]{2}{*}{ Valor $p$} \\
\hline & & $\leq 12$ & $13-18$ & $19-24$ & $>24$ & Habilidades & Não sabem & & \\
\hline \multicolumn{10}{|l|}{ Paridade } \\
\hline 1 & 1529 & 16,5 & 49,7 & 24,6 & 2,1 & 5,6 & 1,6 & 41,22 & $<0,001 \dagger$ \\
\hline 2 & 1006 & 14,8 & 50,5 & 27,0 & 2,2 & 5,0 & 0,8 & & \\
\hline 3 & 626 & 15,6 & 50,9 & 25,3 & 1,4 & 6,4 & 0,5 & & \\
\hline$\geq 4$ & 699 & 22,6 & 49,1 & 20,7 & 1,1 & 5,9 & 0,6 & & \\
\hline \multicolumn{10}{|c|}{ Escolaridade mãe (anos) } \\
\hline $0-4$ & 582 & 22,6 & 46,8 & 22,5 & 3,3 & 4,3 & 0,5 & 87,71 & $<0,001 \dagger$ \\
\hline $5-8$ & 1569 & 18,9 & 48,8 & 24,4 & 1,5 & 5,7 & 0,8 & & \\
\hline $9-11$ & 1282 & 15,3 & 54,1 & 22,2 & 1,3 & 6,0 & 1,1 & & \\
\hline$\geq 12$ & 390 & 7,7 & 46,1 & 36,7 & 2,3 & 5,6 & 1,5 & & \\
\hline \multicolumn{10}{|l|}{ Idade mãe (anos) } \\
\hline Adolescente $(<19)$ & 720 & 20,6 & 44,2 & 27,4 & 3,1 & 4,2 & 0,7 & 41,82 & $<0,001 \dagger$ \\
\hline $20-29$ & 1915 & 15,7 & 52,0 & 23,9 & 1,7 & 5,5 & 1,1 & & \\
\hline $30-39$ & 1098 & 15,7 & 50,6 & 24,6 & 1,2 & 6,8 & 1,0 & & \\
\hline$\geq 40$ & 126 & 26,2 & 46,8 & 19,1 & 2,4 & 4,8 & 0,8 & & \\
\hline \multicolumn{10}{|l|}{ Trabalho materno } \\
\hline $\operatorname{sim}$ & 1522 & 15,5 & 50,3 & 24,9 & 1,9 & 6,1 & 1,3 & 7,49 & $0,28 \dagger$ \\
\hline Não & 2336 & 18,0 & 49,8 & 24,4 & 1,8 & 5,3 & 0,8 & & \\
\hline
\end{tabular}

† Qui-quadrado de associação; * Tendência linear. 
observa-se que as mães com escolaridades entre 5 e 11 anos iniciaram o treinamento mais cedo, assim como as adolescentes e as maiores de 40 anos. As mães das crianças que não usavam mais fraldas de dia tinham menor escolaridade, maior número de filhos e mais de 40 anos.

A orientação médica sobre o controle esfincteriano foi extremamente baixa no conjunto $(2,2 \%)$, apresentando uma tendência crescente de orientação com nível econômico. Nas mães do primeiro quintil ela foi pequena $(1 \%)$, e mesmo no quintil mais rico limitou-se a $5,6 \%$.

A expectativa de retirar fraldas (Tabela 5) antes dos 12 meses foi maior entre as mães com menor escolaridade, maiores de 40 anos e com maior número de filhos.

\section{Discussão}

Este é o primeiro estudo brasileiro a avaliar o controle esfincteriano em crianças saudáveis, ainda durante o processo de aquisição do controle. A realização deste trabalho no contexto de um estudo longitudinal permitirá o monitoramento desse processo ao longo do tempo. Esta primeira avaliação foi realizada quando as crianças da Coorte de Nascimento de Pelotas, 2004, completaram 12 meses de idade. Essa abordagem permitiu coletar as informações de interesse no momento em que ocorreram, minimizando o viés de memória. As perdas de acompanhamento nesta primeira etapa foram bastante reduzidas $(4,9 \%)$. As próximas etapas planejadas do estudo são visitas quando as crianças completarem 24 e 48 meses de idade.

O controle esfincteriano é um desafio para os pais e sua aquisição um marcador do adequado desenvolvimento infantil. A Academia Americana de Pediatria recomenda que o treinamento esfincteriano seja iniciado por volta dos 18 meses, quando a maioria das crianças estão fisiológica e psicologicamente aptas para este treinamento. 6 Entretanto, Brazelton et al.21 ressaltam a importância da avaliação das habilidades e do desejo da criança para que se defina o momento ideal do início desse treinamento. Apenas $5,6 \%$ das mães entrevistadas em nosso estudo relataram habilidades da criança como fator importante para o início do treinamento.

$\mathrm{O}$ presente estudo demonstrou um pequeno número de crianças sem fraldas durante o dia $(1,3 \%)$, valor próximo aos $2 \%$ relatados por Martin et al. 22
Isso não permitiu avaliar diferenças entre os sexos em relação à aquisição do controle esfincteriano que, conforme relatos de literatura é mais precoce nas meninas. 23 Por outro lado, $14,7 \%$ das mães já tinham iniciado a retirada das fraldas de seus filhos antes dos 12 meses. Essa freqüência elevada de início precoce do treinamento reflete a expectativa da maioria das mães de que seus filhos deixem as fraldas antes dos 18 meses, expectativa irreal, que também foi descrita em outros estudos. 8,24

Por outro lado, a literatura sugere que a expectativa de uma retirada muito precoce das fraldas está sendo substituída por maior tolerância, com conseqüente retardamento da idade de abandono das fraldas. Uma recente revisão da literatura revela que a idade média de aquisição de controle esfincteriano passou de aproximadamente 24 meses nos anos 50 para 39 meses nos anos 90.23

Olson et al.,25 em estudo com pais e pediatras, nos Estados Unidos, sobre as orientações preventivas em consultas de rotina, relataram que os tópicos mais discutidos pelos médicos durante os primeiros três anos de vida são imunizações, amamentação, alimentação, sono e transporte seguro de crianças. Entre 11 tópicos citados por pais e pediatras, para crianças na faixa etária dos 10 aos 18 meses, o treinamento de esfíncteres foi o menos discutido (17\%). No presente estudo, uma parcela muito menor de mães foi orientada pelo pediatra $(2,2 \%)$. Mesmo entre as mães mais ricas, o grupo que mais recebeu orientação $(5,6 \%)$, a prevalência de orientação ficou muito abaixo do estudo americano.

Assim, mães não orientadas pelos pediatras, e com uma expectativa por demais precoce em relação à idade da retirada das fraldas, acabam por iniciar o treinamento dos filhos antes do momento ideal, o que leva com freqüência a insucessos e frustrações. ${ }^{26,27}$ Além disso, a incidência de disfunção miccional vem aumentando nos últimos anos e existe a suspeita de que estratégias inadequadas de treinamento esfincteriano possam estar associadas a tais disfunções. 15

Fica evidente a necessidade de que o pediatra tome para si a responsabilidade da orientação sobre o treinamento de controle de esfíncteres, hoje por conta das avós, amigas e atendentes de creche. A orientação informada do pediatra pode, potencialmente, reduzir tanto a frustração causada por uma tentativa mal sucedida de retirada de fraldas, como possíveis futuras disfunções das eliminações. 


\section{Referências}

1. Muellner SR. Development of urinary control in children: a new concept in cause, prevention and treatment of primary enuresis. J Urol. 1960; 84: 714-6.

2. Gesell A. The ontogenesis of infant behavior. In: Carmichael L, editor. Manual of child psycology. New York: John Wilty; 1954.

3. Oppel WC, Harper PA, Rider RV. The age of attaining bladder control. Pediatrics. 1968; 42: 614-26.

4. deVries MW, deVries MR. Cultural relativity of toilet training readiness: a perspective from East Africa. Pediatrics. 1977; 60: 170-7. )

5. Horner MM, McClellan MA. Toilet training: ready or not? Pediatr Nurs. 1981; 7: 15-8.

6. Stadtler AC, Gorski PA, Brazelton TB. Toilet training methods, clinical interventions, and recommendations of American Academy of Pediatrics. Pediatrics. 1999; 103: 1359-68.

7. Doleys DM, Dolce JJ. Toilet training and enuresis. Pediatr Clin North Am. 1982; 29: 297-313.

8. Stehbens JA, Silber DL. Parental expectations in toilet training. Pediatrics. 1971; 48: 451-4.

9. Brazelton TB. A child-oriented approach to toilet training. Pediatrics. 1962; 29: 121-8.

10. Hellstrom AL, Sillen U. Early potty training advantageous in bladder dysfunction. Decreases the risk of urinary infection. Lakartidningen. 2001; 98: 3216-9.

11. Hellstrom AL. Influence of potty training habits on dysfunctional bladder in children. Lancet. 2000; 356: 1787.

12. Von Gontard A. Elimination disorders in childhood. How to make children dry and clean. MMW Fortschr Med. 2003; 145: 26-30.

13. De Paepe H, Renson C, Hoebeke P, Raes A, Van Laecke E, Vande Walle J. The role of pelvic-floor therapy in the treatment of lower urinary tract dysfunctions in children. Scand J Urol Nephrol. 2002; 36: 260-7.

14. De Paepe H, Renson C, Van Laecke E, Raes A, Vande Walle J, Hoebeke P. Pelvic-floor therapy and toilet training in young children with dysfunctional voiding and obstipation. BJU Int. 2000; 85: 889-93.

15. Bakker E, Wyndaele JJ. Changes in the toilet training of children during the last 60 years: the cause of an increase in lower urinary tract dysfunction? BJU Int. 2000; 86: 248-52.
16. Bakker E, van Gool J, van Sprundel M, van der Auwera JC, Wyndaele JJ. Risk factors for recurrent urinary tract infection in 4,332 Belgian schoolchildren aged between 10 and 14 years. Eur J Pediatr. 2004; 163: 234-8.

17. Barros AJ, Santos Ida S, Victora CG, Albernaz EP, Domingues MR, Timm IK, Matijasevich A, Bertoldi AD, Barros FC. The 2004 Pelotas birth cohort: methods and description. Rev Saúde Pública. 2006; 40: 402-13.

18. Newborg J, Stock J, Wnek L, Guidabaldi JJS. Battelle developmental inventory: Itasca, I11: Riverside; 1988.

19. Frankenburg K, Dodds J, Archer P, Bresnick B. Denver II: tecnical manual and training manual. Denver: Denver Developmental Materials; 1990.

20. Barros AJ, Victora CG. A nationwide wealth score based on the 2000 Brazilian demographic census. Rev Saúde Pública. 2005; 39: 523-9.

21. Brazelton TB, Christophersen ER, Frauman AC, Gorski PA, Poole JM, Stadtler AC, Wright CL. Instruction, timeliness, and medical influences affecting toilet training. Pediatrics. 1999; 103: 1353-8.

22. Martin JA, King DR, Maccoby EE, Jacklin CN. Secular trends and individual differences in toilet-training progress. J Pediatr Psychol. 1984; 9: 457-67.

23. Schum TR, Kolb TM, McAuliffe TL, Simms MD, Underhill RL, Lewis M. Sequential acquisition of toilet-training skills: a descriptive study of gender and age differences in normal children. Pediatrics. 2002; 109: E48

24. Pachter LM, Dworkin PH. Maternal expectations about normal child development in four cultural groups. Arch Pediatr Adolesc Med. 1997; 151: 1144-50.

25. Olson LM, Inkelas M, Halfon N, Schuster MA, O'Connor $\mathrm{KG}$, Mistry R. Overview of the content of health supervision for young children: reports from parents and pediatricians. Pediatrics. 2004; 113 (6 Suppl): 1907-16.

26. Chamberlin RW. Prevention of behavioral problems in young children. Pediatr Clin North Am. 1982; 29: 239-47.

27. Rapoff MA, Christophersen ER. Improving compliance in pediatric practice. Pediatr Clin North Am. 1982; 29: 33957.

Recebido em 8 de dezembro de 2006

Versão final apresentada em 6 de setembro de 2007

Aprovado em 23 de outubro de 2007 